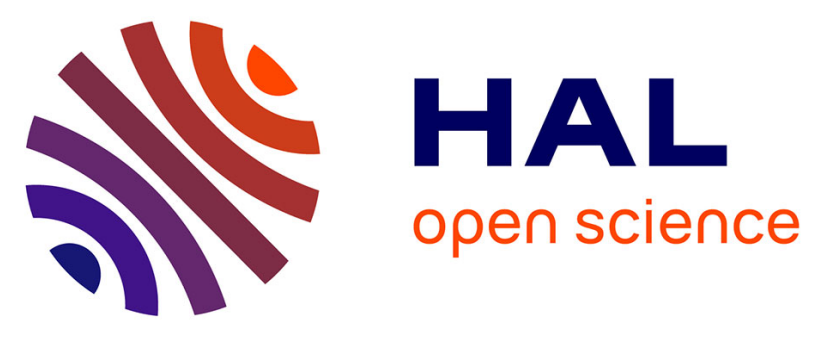

\title{
Spin Thermometry: a Straightforward Measure of Millikelvin Deuterium Spin Temperatures Achieved by Dynamic Nuclear Polarization
}

Behdad Aghelnejad, Sina Marhabaie, Mathieu Baudin, Geoffrey

Bodenhausen, Diego Carnevale

\section{To cite this version:}

Behdad Aghelnejad, Sina Marhabaie, Mathieu Baudin, Geoffrey Bodenhausen, Diego Carnevale. Spin Thermometry: a Straightforward Measure of Millikelvin Deuterium Spin Temperatures Achieved by Dynamic Nuclear Polarization. Journal of Physical Chemistry Letters, 2020, 11 (9), pp.3219-3225. 10.1021/acs.jpclett.0c00713 . hal-02877216

\section{HAL Id: hal-02877216 https://hal.sorbonne-universite.fr/hal-02877216}

Submitted on 22 Jun 2020

HAL is a multi-disciplinary open access archive for the deposit and dissemination of scientific research documents, whether they are published or not. The documents may come from teaching and research institutions in France or abroad, or from public or private research centers.
L'archive ouverte pluridisciplinaire HAL, est destinée au dépôt et à la diffusion de documents scientifiques de niveau recherche, publiés ou non, émanant des établissements d'enseignement et de recherche français ou étrangers, des laboratoires publics ou privés. 


\title{
Spin Thermometry: a Straightforward Measure of Millikelvin Deuterium Spin Temperatures Achieved by Dynamic Nuclear Polarization
}

\author{
Behdad Aghelnejad, ${ }^{1,2}$ Sina Marhabaie, ${ }^{1,3}$ Mathieu Baudin, ${ }^{1,4}$ Geoffrey Bodenhausen ${ }^{1}$ and Diego \\ Carnevale ${ }^{1, *}$ \\ ${ }^{1}$ Laboratoire des biomolécules, LBM, Département de chimie, École normale supérieure, PSL University, Sorbonne \\ Université, CNRS, 75005 Paris, France \\ ${ }^{2}$ Bruker Biospin SAS, F-67160 Wissembourg, France \\ ${ }^{3}$ IFSTTAR, Université Paris-Est, UPEMLV, Marne-la-Vallée, France \\ ${ }^{4}$ Université de Paris, Laboratoire de Chimie et Biologie Pharmacologiques et Toxicologiques, CNRS UMR 8601, \\ Université Paris Descartes, 45 rue des Saints Pères 75006 Paris France
}

Accepted on J Phys Chem Lett

https://doi.org/10.1021/acs.jpclett.0c00713

Corresponding author: Diego Carnevale

diego.carnevale@ens.fr 


\section{Abstract}

Dynamic nuclear polarization of samples at low temperatures, typically between 1.2 and $4.2 \mathrm{~K}$, allows one to achieve spin temperatures as low as $2 \mathrm{mK}$, so that for many nuclear isotopes the high-temperature approximation is violated for the nuclear Zeeman interaction. This leads to characteristic asymmetries in powder spectra. We show that the lineshapes due to the quadrupolar couplings of deuterium spins present in virtually all solvents used for such experiments ('DNP juice') allows the quick yet accurate determination of the deuterium spin temperature or, equivalently, the deuterium polarization. The observation of quadrupolar echoes excited by small flip-angle pulses allows one to monitor the build-up and decay of the positive or negative deuterium polarization.
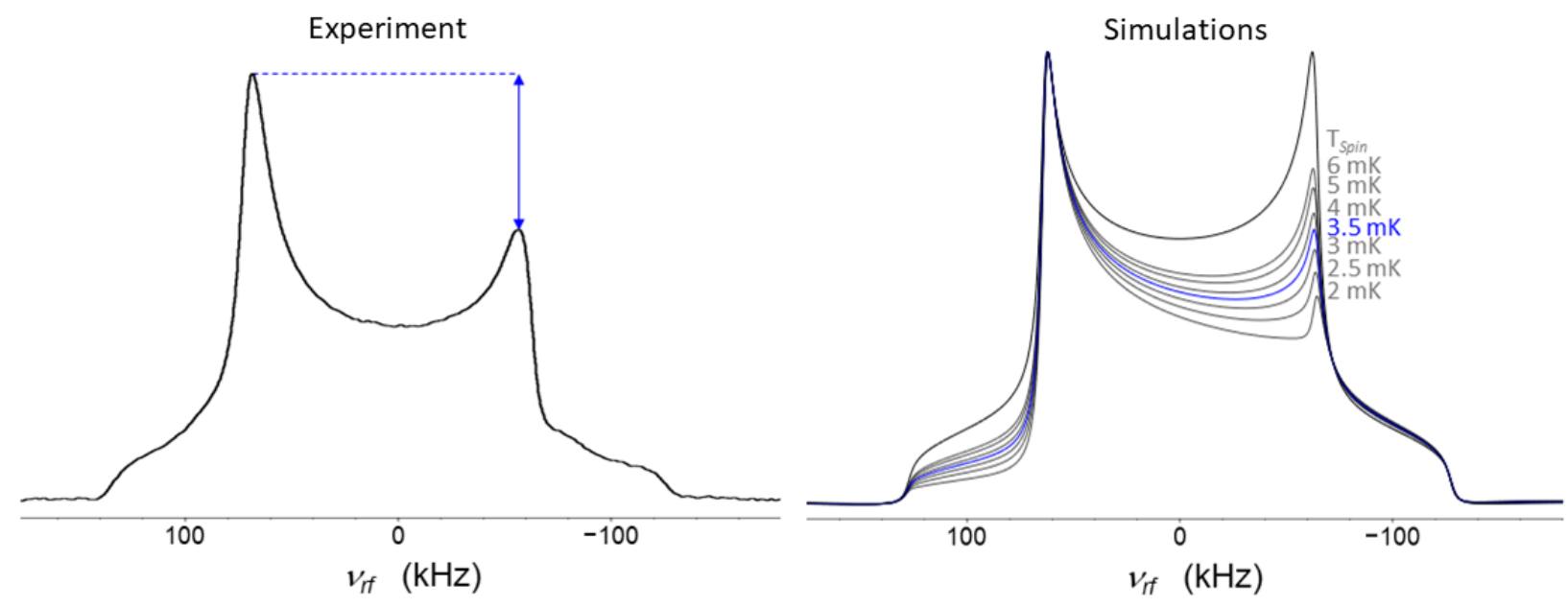

\section{Key words}

Dynamic Nuclear Polarization. Spin Temperature. Pake Pattern. Quadrupolar Coupling. 
Dissolution Dynamic Nuclear Polarization (D-DNP) allows one to boost the sensitivity of magnetic resonance by about four orders of magnitude: ${ }^{1-5}$ roughly two orders of magnitude can be gained by lowering the sample temperature from 300 to ca. $3 \mathrm{~K}$, and two further orders of magnitude can be gained by partially saturating the EPR transitions of polarizing agents (radicals) such as TEMPOL (4-hydroxy-2,2,6,6-tetramethylpiperidin-1-oxyl) by microwave saturation of their ESR transitions. In a static field of $B_{0}=6.7 \mathrm{~T}$, the polarization of protons can be boosted to $P\left({ }^{1} \mathrm{H}\right)>90 \%$, while cross-polarization ${ }^{6-11}$ from abundant protons to dilute nuclei such as ${ }^{13} \mathrm{C}$ can yield polarizations $P\left({ }^{13} \mathrm{C}\right)>70 \%$ in ca. 10 minutes, corresponding to a spin temperature $T_{\text {spin }}\left({ }^{13} \mathrm{C}\right)<2 \mathrm{mK}$. The hyperpolarized samples can be rapidly dissolved ${ }^{4,5}$ and transferred to NMR or MRI systems. Suitable precautions must be taken to preserve the polarization. ${ }^{12,13}$

To optimize D-DNP experiments, the nuclear spin polarization $P(S)$, or, equivalently, the spin temperature $T_{\text {spin }}(S)$, must be determined twice, both before and after dissolution, ideally by independent methods. Prior to dissolution, this is usually done by comparing the signal intensities of protons or low-gamma nuclei $S$ obtained with and without DNP for $1.2<T_{\text {sample }}<$ $4.2 \mathrm{~K}$, where the spectra of amorphous frozen solids are very broad, typically $100 \mathrm{kHz}$ for ${ }^{1} \mathrm{H}$ and ${ }^{2} \mathrm{H}$, and $50 \mathrm{kHz}$ for ${ }^{13} \mathrm{C}$.

After dissolution, one can likewise compare signal intensities of protons or low-gamma nuclei $S$ in solution, either boosted by DNP or obtained at thermal equilibrium after complete relaxation. The latter signals are many orders of magnitude weaker than the former, so that extensive signal averaging is often required. A more efficient alternative consists in measuring the asymmetries of doublets due to homo- or heteronuclear scalar couplings after dissolution. ${ }^{14-17}$

This work presents an alternative approach to determine the deuterium polarization $P\left({ }^{2} \mathrm{H}\right)$ (or equivalently, the deuterium spin temperature $\left.T_{\text {spin }}\left({ }^{2} \mathrm{H}\right)\right)$ in the frozen solid prior to dissolution, by detecting the asymmetry of quadrupolar doublets of deuterons in partly deuterated samples. Following widespread practice, our frozen sample ("DNP juice") contains $60 \%$ ethanol- $d_{6}, 30 \%$ $\mathrm{D}_{2} \mathrm{O}, 10 \% \mathrm{H}_{2} \mathrm{O}$ and $40 \mathrm{mM}$ TEMPOL. Ethanol- $\mathrm{d}_{6}$, glycerol- $\mathrm{d}_{8}$, or DMSO-d $\mathrm{d}_{6}$ prevent the formation of ice crystals that are deleterious for DNP. Note that all deuterated constituents of such frozen 
mixtures have roughly the same quadrupolar splitting. Methyl groups do not rotate freely below 4.2 $\mathrm{K}$ (although methyl groups can undergo fast rotational tunneling in some cases such as gamma-picoline) ${ }^{18}$ so that the quadrupolar couplings of most deuterated methyl groups are not reduced by averaging. There is therefore no need to add any specific molecules that contain deuterium.

Because of extensive dipole-dipole couplings, the proton spectra of such amorphous frozen solids have broad shapes with linewidths on the order of $100 \mathrm{kHz}$. These lineshapes can become asymmetric at very low spin temperatures, and the sign of the asymmetry changes with the sign of the spin temperature, e.g., after inversion by a $180^{\circ}$ pulse ${ }^{19,20}$ However, it appears difficult to determine the precise spin temperature from the analysis of the asymmetry of such broad lines. ${ }^{19,21-23}$ Marohn et al. ${ }^{24}$ detected asymmetric quadrupolar satellites in optically detected ${ }^{71} \mathrm{Ga} N \mathrm{NR}$ and could assess the polarization level of its nuclear spin $S=3 / 2$ from the asymmetry of the satellites. On the other hand, well-resolved dipolar Pake patterns can be observed in ${ }^{1} \mathrm{H}$ NMR of water molecules that are isolated in suitable matrices such as crystals of barium chlorate monohydrate. ${ }^{25-28}$ As we shall discuss in a separate paper, the spin temperature of the protons in such isolated water molecules can be lowered by DNP after suspending the crystallites in a solution of radicals prior to freezing.

It has recently been observed ${ }^{29}$ that deuterium spectra of amorphous frozen solids reveal Pakelike powder patterns determined by the quadrupole interaction. In a static field of $B_{0}=6.7 \mathrm{~T}$, at spin temperatures $T_{\text {spin }}\left({ }^{2} \mathrm{H}\right)$ below ca. $10 \mathrm{mK}$, these patterns are asymmetrical because of the violation of the high-temperature approximation, provided that the coherences are excited with small flip angle pulses. As we shall demonstrate in this Letter, the asymmetry of the Pake-like powder patterns allows one to determine the spin temperature.

The asymmetry of such ${ }^{2} \mathrm{H}$ spectra is reminiscent of similar effects observed in frozen doubly ${ }^{15} \mathrm{~N}$-labelled $\mathrm{N}_{2} \mathrm{O} \cdot{ }^{30}$ The ${ }^{15} \mathrm{~N}$ spectra feature four partially overlapping Pake-like patterns that depend not only on the dipole-dipole couplings between the two ${ }^{15} \mathrm{~N}$ nuclei, but also on the CSA tensors and their relative orientations. A careful analysis allowed the determination of an absolute polarization $P\left({ }^{15} \mathrm{~N}\right)=10.2 \%$ after $37 \mathrm{~h}$ of microwave irradiation, without recording a 
time-consuming spectrum in thermal equilibrium. ${ }^{30}$ An alternative method has been proposed ${ }^{31}$ to measure the polarization $P\left({ }^{1} \mathrm{H}\right)$ by monitoring separately the Zeeman order $S_{z}$ and the twospin order $2 I_{z} S_{Z}\left(I={ }^{1} \mathrm{H}, S={ }^{13} \mathrm{C}\right)$ under magic-angle spinning conditions at $35 \mathrm{~K}<T_{\text {sample }}<45 \mathrm{~K}$.

Our method based on the observation of ${ }^{2} \mathrm{H}$ spectra of frozen deuterated mixtures only depends on the quadrupolar coupling that is roughly the same for most frozen solvents. The asymmetry of the ${ }^{2} \mathrm{H}$ spectra discussed in this Letter is reminiscent of the work by Andersen et al., ${ }^{32}$ who compared the intensities of two lines in nuclear quadrupole resonance (NQR) of ${ }^{127}$ I that can be split by a weak Zeeman effect.

The use of $r f$-pulses with small flip angles is dictated by the necessity to preserve the hyperpolarization as it builds up under microwave irradiation. ${ }^{19,23,33}$ Small flip angle pulses have revealed asymmetries in proton spectra at very low spin temperatures. ${ }^{20,23}$ It is well known that the use of $90^{\circ}$ pulses completely destroys the hyperpolarization. We performed numerical simulations of these effects on a first-order quadrupolar pattern associated with ${ }^{2} H(\operatorname{spin} I=1)$, which is common constituent of 'DNP juice'. The simulated spectra shown in Figure 1(a) were obtained by Fourier transformation of free induction decays excited by ideal excitation pulses with the $r f$ carrier placed in the center of the quadrupolar doublets, assuming that receiver could be activated immediately after an $r f$ pulse. The nutation angle $\beta$ was incremented from 9 to $171^{\circ}$ in steps of $9^{\circ}$ (from bottom to top). The initial state corresponds to a fully polarized $I=1$ spin, where only the $|1,+1\rangle$ state is populated $\left(T_{\text {spin }}\left({ }^{2} \mathrm{H}\right)=0 \mathrm{~K}\right)$. For $\beta<20^{\circ}$ one obtains only $|1,+1\rangle \leftrightarrow|1,0\rangle$ coherences, so that the powder pattern comprises only one of the two "lobes" or "cusps" of the Pake pattern. When the flip angle approaches $180^{\circ}$, one obtains only the complementary lobe that is due to $|1,-1\rangle \leftrightarrow|1,0\rangle$ coherences. As expected, a symmetrical Pake pattern is obtained when $\beta=90^{\circ}$, due to the uniform excitation of both single-quantum coherences, regardless of the initial population distribution. At the same time, a $90^{\circ}$ pulse leads to the equalization of the populations of all three spin states and therefore destroys the hyperpolarization. Figure 1 (b) shows the density matrices and energy level diagrams corresponding to the single-transition spectra shown in blue in Fig. 1(a). The quadrupolar 
parameters for ${ }^{2} \mathrm{H}$ were assumed to be $C_{Q}=170 \mathrm{kHz}$ and $\eta_{Q}=0 .{ }^{34}$ The ${ }^{2} \mathrm{H}$ chemical shift anisotropy was ignored for simplicity.

Figure 2 shows experimental ${ }^{2} \mathrm{H}$ spectra obtained with quadrupolar echoes ${ }^{34,35}$ excited by a $18^{\circ}$ $\tau$ - $18^{\circ}-\tau$ - sequence combined with phase-cycling in the manner of Exorcycle ${ }^{36}$ in order to select the coherence transfer pathway ${ }^{37} p=0 \rightarrow p=+1 \rightarrow p=-1$. Although such small-angle quadrupolar echoes do not achieve complete refocusing of either linear or quadratic inhomogeneities - which is best achieved by using a refocusing pulse ${ }^{24,25}$ with $\beta=90^{\circ}-$ they result nonetheless in reasonably undistorted lineshapes that are much easier to interpret than those produced by a pulse-acquire approach. ${ }^{26}$ Furthermore, and more importantly, echoes allow one to remove possible background signals - which are particularly detrimental when observing ${ }^{1} \mathrm{H}$ - and remove distortions due to acoustic ringing of the coil. ${ }^{27,28,38,39}$ The use of spin echoes has proven successful for the acquisition of undistorted inhomogeneous lineshapes in static solid samples, even when only very weak $r f$-field strengths are available. ${ }^{26}$ The black spectrum in Fig. 2 was acquired without microwave irradiation at $T_{\text {spin }}=T_{\text {sample }}=1.3 \mathrm{~K}$ (in liquid helium at $158 \mathrm{~Pa}$ ) and results in a symmetric quadrupolar pattern. After the microwave irradiation is switched on, a progressive asymmetry of the lineshape builds up, revealing the lowering of the spin temperature $\left(T_{\text {spin }}<<T_{\text {sample }}\right)$, or, equivalently, the build-up of a hyperpolarized state. As expected, the signal-to-noise ratio also improves as $T_{\text {spin }}$ decreases. The highest polarization achieved in this series is shown in blue $\left(T_{\text {spin }}=3.5 \mathrm{mK}\right)$ whereas spectra obtained at intermediate spin temperatures are shown in grey.

Our sample contains a variety of deuterated groups like $C D_{3}, C D_{2}$ and $O D$ of ethanol- $d_{6}(60 \%)$ and $\mathrm{D}_{2} \mathrm{O}(30 \%)$. Nonetheless, a single well-defined Pake pattern is obtained in these experiments, indicating that, in the absence of motional narrowing, the quadrupolar parameters are very similar in different molecular environments, at least within the homogeneous linewidth of ca. $7 \mathrm{kHz}$. This is also true for the isotropic chemical shifts and isotropic parts of the secondorder quadrupole coupling of ${ }^{2} \mathrm{H}$ that typically span ca. $10 \mathrm{ppm}$ (ca. $0.44 \mathrm{kHz}$ at $B_{0}=6.7 \mathrm{~T}$ ). ${ }^{40}$ Numerical fits of the ${ }^{2} \mathrm{H}$ lineshape acquired without microwave irradiation produced $C_{Q}=170$ $\mathrm{kHz}, \eta_{Q}=0$ and a line broadening of $7 \mathrm{kHz}$, in remarkable agreement with those of isolated ${ }^{2} \mathrm{H}_{2} \mathrm{O}$ 
molecules trapped in solid barium chlorate reported by Long et al. ${ }^{34}$ The broadening may be due in part to homo- and heteronuclear dipolar couplings.

The Boltzmann polarization $P(I)$ of a nuclear spin $I$ with a spin temperature $T_{\text {spin }}(I)$ is usually defined as:

$$
P(I)=\tanh \left[\hbar \gamma_{I} B_{0} /\left(2 k_{B} T_{\text {spin }}(I)\right)\right]
$$

where $\gamma_{1}$ is the gyromagnetic ratio and $1 /\left(k_{B} T_{I}\right)$ is the inverse spin temperature. If these populations obey a Boltzmann distribution, as they should when in equilibrium with a thermal reservoir at a spin temperature $T_{\text {spin }}(I)$, the density operator $\rho_{\text {eq }}$ for a spin $I=1$, neglecting the unobservable identity operator, can be concisely expressed as $\rho_{e q}=P(I) I_{z}+P(I)^{2} I_{z}^{2}$ :

$$
\rho_{e q}=\tanh \left[\hbar \gamma_{I} B_{0} /\left(2 k_{B} T_{\text {spin }}(I)\right)\right] I_{z}+\tanh ^{2}\left[\hbar \gamma_{I} B_{0} /\left(2 k_{B} T_{\text {spin }}(I)\right)\right] I_{z}^{2} .
$$

This density operator can be considered as initial condition for simulations of any spin dynamics by means of the usual Liouville-von Neumann equation $\rho(t)=U(t) \rho(0) U(t)^{-1}$, with $\rho(0)=\rho_{e q}$ for any arbitrary spin temperature.

Figure 3(a) shows an experimental hyperpolarized ${ }^{2} \mathrm{H}$ spectrum analogous to Fig. 2 . The striking asymmetry of the two horns of the pattern is highlighted by a blue arrow. Figure 3(b) shows a series of spectra simulated for different values of the polarization $P\left({ }^{2} \mathrm{H}\right)$, assuming $18^{\circ}-\tau-18^{\circ}-\tau$ quadrupolar echoes with realistic rectangular $r f$ pulses $\left(v_{1}=50 \mathrm{kHz}\right)$ combined with Exorcycle. ${ }^{36}$ The simulated spectrum that best reproduces the asymmetry observed in (a) is shown in blue, corresponding to a spin temperature $T_{\text {spin }}\left({ }^{2} \mathrm{H}\right)=3.5 \mathrm{mK}$, which amounts to a polarization $P\left({ }^{2} \mathrm{H}\right)=$ $29 \%$. These simulations show that the absolute deuterium spin temperature and polarization can be determined with an accuracy of ca. $\pm 0.5 \mathrm{mK}$ and $\pm 4 \%$, respectively. Clearly, the use of echoes with small flip-angle pulses allows one to acquire undistorted deuterium lineshapes of hyperpolarized samples. If the conditions for thermal mixing are fulfilled, different isotopes should have the same spin temperature, e.g., $T_{\text {spin }}\left({ }^{13} \mathrm{C}\right)=T_{\text {spin }}\left({ }^{2} \mathrm{H}\right)$, provided one does not employ cross polarization. Thermal mixing should prevail if the target molecules dissolved in the DNP 
juice are isotopically enriched in ${ }^{13} \mathrm{C}$ to facilitate ${ }^{13} \mathrm{C}-{ }^{13} \mathrm{C}$ spin diffusion and if the ${ }^{13} \mathrm{C}$ nuclei are abundant enough so that the electron-spin couplings are efficient. ${ }^{41}$ In contrast to other DNP mechanisms which can be described by isolated electron-nucleus spin pairs (Overhauser and solid effects) or by a system comprising two electrons and one nucleus (cross effect), thermal mixing is more conveniently described by a thermodynamic model. ${ }^{42}$ This occurs for high radical concentrations so that dipolar electron-electron couplings broaden the EPR lineshape homogeneously. The TEMPOL concentration of $40 \mathrm{mM}$ utilized in this study is similar to the doping used by Guarin et al. ${ }^{41}$ for investigations of DNP enhancements in the thermal mixing regime.

In order to extract the spin temperature $T_{\text {spin }}$ from a lineshape analysis of such asymmetric deuterium spectra, a numerical fit assuming quadrupolar echoes $\beta$ - $\tau$ - $\beta$ - $\tau$ - combined with Exorcycle acting on $\rho_{\text {eq }}\left(T_{\text {spin }}\right)$ of Eq. 2 needs to be performed. This ultimately means that the main parameter to be optimised is $\rho_{e q}\left(T_{\text {spin }}\right)$. Other parameters such as the quadrupolar coupling constant $C_{Q}$, the asymmetry $\eta_{Q}$ and homogeneous linewidth can be easily estimated from symmetric lineshapes obtained with similar $90^{\circ}-\tau$ - $90^{\circ}-\tau$ - echoes on the same sample, either with or without DNP. Figure 4 shows that a more straightforward estimate of the spin temperature may be obtained by simply evaluating the ratio of the peak heights of the two 'horns' of the Pake pattern. The asymmetry of the quadrupolar powder pattern can be expressed by the ratio $\left(I_{\max }-I_{\min }\right) / I_{\max }$, where $I_{\max }$ and $I_{\min }$ are the peak heights of the high- and low-frequency 'horns'. The ratios shown in Fig. 4 were obtained by varying one parameter at the time while leaving all others at their optimal value. The asymmetry of the peak heights in the experimental spectrum of Fig. $3 a$ is indicated by a horizontal dashed line in Fig. 4 . The parameters $C_{Q}$ and $\eta_{Q}$ can be easily estimated prior to DNP as discussed above; they not only affect the asymmetry of the two wings of the powder pattern but also alter the splitting between the horns. The rf-field amplitude and the homogeneous linewidth have little effect on the estimates of $T_{\text {spin. }}$. The (conservative) estimate $\pm 0.5 \mathrm{mK}$ of the confidence range is indicated by horizontal dashed lines.

Figure 5 shows typical build-up profiles of the integrals of ${ }^{2} \mathrm{H}$ signals under microwave irradiation. A positive enhancement is induced by saturation of the low-frequency lobe of the 
EPR line of TEMPO at $187.9 \mathrm{GHz}$, i.e., of the part of the EPR spectrum that upon saturation leads to a positive enhancement of the nuclear transitions. The top panel of Fig. 4 indicates how the microwave frequency and amplitude are switched. When the frequency $v_{\mu \mathrm{w}}$ drops to zero, this means that the microwave source is turned off. A single echo excited by a $18^{\circ}-\tau$ - $18^{\circ}-\tau$ - echo sequence was acquired in each case, since a complete four-step phase cycle cannot be carried out while the polarization builds up. As a result, ideal refocusing of quadrupolar and shift interactions was not achieved in these spectra, thus resulting in somewhat distorted patterns. Nonetheless, the integrals over the powder patterns suffice for the purpose of monitoring the evolution of the hyperpolarization as it builds up over time. The subsequent interruption of the microwave irradiation leads to a loss of hyperpolarization due to a gradual return to the

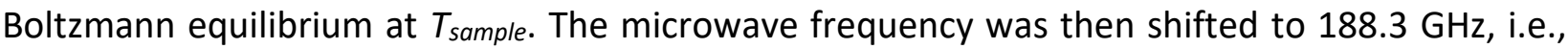
to the high-frequency lobe of the EPR line, resulting in cooling towards negative spin temperatures. Profiles acquired at $T_{\text {sample }}=1.3$ and $4.0 \mathrm{~K}$, combined with frequency switching at different times to take the different time scales of the build-up curves into account, are shown by continuous and dashed black lines, respectively. The gain in signal-to-noise obtained by lowering the temperature of the sample is evident.

In conclusion, we have investigated the quadrupolar lineshapes due to ${ }^{2} \mathrm{H}$ nuclei in a typical frozen DNP juice. The asymmetry of experimental hyperpolarized powder patterns, observed in the presence of microwave irradiation by means of small flip-angle quadrupolar echoes combined with phase-cycling, is in very good agreement with simulations. Quadrupolar echoes excited by small flip-angle pulses do not destroy the hyperpolarization and yield undistorted powder patterns in hyperpolarized solid samples. The comparison of experimental and simulated lineshapes allows one to determine the absolute spin polarization or, equivalently, the absolute spin temperature of the deuterium nuclei. If the conditions of thermal mixing are fulfilled, the spin temperatures of different nuclei such as ${ }^{6} \mathrm{Li},{ }^{13} \mathrm{C},{ }^{15} \mathrm{~N},{ }^{31} \mathrm{P}$, etc., should be equal to the spin temperature $T_{\text {spin }}\left({ }^{2} \mathrm{H}\right)$ of the deuterium nuclei. 


\section{Experimental Details}

All experiments were conducted in a $B_{0}=6.7 \mathrm{~T}$ prototype polarizer designed by Bruker with Larmor frequencies $v_{0}\left({ }^{1} \mathrm{H}\right)=285.3 \mathrm{MHz}$ and $v_{0}\left({ }^{2} \mathrm{H}\right)=43.8 \mathrm{MHz}$. A home-built broadband double

resonance ${ }^{1} \mathrm{H}-\mathrm{X}$ D-DNP probe ${ }^{29}$ was used where the $\mathrm{X}$ channel can cover a range of nuclei from ${ }^{15} \mathrm{~N}$ to ${ }^{23} \mathrm{Na}$, i.e., from 28.9 to $75.5 \mathrm{MHz}$ at $6.7 \mathrm{~T}$, without removing the probe from the cryostat. This probe uses a horizontal solenoidal coil to generate a homogeneous rf $B_{1}$ field. Unlike most conventional setups that have at least a few capacitors outside the polarizer for the fine adjustment of tuning and matching, the complete $r f$ circuit is immersed in the low-temperature cryostat inside the polarizer.

The sample ("DNP juice") of $300 \mu$ l contained $60 \%$ ethanol- $\mathrm{d}_{6}, 30 \% \mathrm{D}_{2} \mathrm{O}, 10 \% \mathrm{H}_{2} \mathrm{O}$ and $40 \mathrm{mM}$ TEMPOL. The microwaves are provided by an ELVA-1 source coupled to a Virginia Diodes (VDI) frequency doubler. Frequency modulation was achieved with a saw-tooth waveform with a modulation frequency of $1 \mathrm{kHz}$ to cover a bandwidth of $200 \mathrm{MHz}$ to saturate a significant fraction of the EPR spectrum. For positive polarization, the central microwave frequency was set to $187.9 \mathrm{GHz}$. For negative polarization, the central frequency was set to $188.3 \mathrm{GHz}$ while all other parameters were kept constant.

The $r f$ carrier was set in the center of the quadrupolar powder pattern. The $r f$-field amplitude for deuterium pulses was $v_{1}=50 \mathrm{kHz}$, corresponding to flip angles $\beta=18,27$ and $36^{\circ}$ for pulse lengths $\tau_{p}=1,1.5$ and $2 \mu \mathrm{s}$, respectively. With the exception of the build-up curves of Fig. 4, where only single echoes were acquired, the $\beta$ - $\tau$ - $\beta$ - $\tau$ - echoes were phase cycled to select the $p=$ $0 \rightarrow p=+1 \rightarrow p=-1$ coherence pathway ${ }^{37}$ using a four-step phase cycle ${ }^{36}$ so as to i) eliminate acoustic ringing and ii) refocus both linear shielding and quadratic first-order quadrupolar interactions that would otherwise result in distorted lineshapes. ${ }^{43}$ The refocusing time was $\tau=$ $15 \mu \mathrm{s}$ and delays between subsequent echo sequences were varied between 30 and $500 \mathrm{~s}$. A train of 100 pulses was applied to saturate the ${ }^{2} \mathrm{H}$ nuclei with $\tau_{p}=5 \mu \mathrm{s}\left(\beta=90^{\circ}\right)$ spaced by $100 \mu \mathrm{s}$ prior to all experiments. 
All simulations of NMR spectra were calculated with Simpson ${ }^{44}$ and involved powder averaging over 4180 crystallite orientations sampled with the ZCW scheme. ${ }^{45-47}$ Ideal pulses were assumed in the simulations of Fig. 1 whereas rectangular pulses with finite $r f$ amplitudes were utilized for the simulations of the exorcycled echoes of Fig. 3. An input file is given in Supporting Information.

\section{Acknowledgements}

This work was supported by the CNRS, the European Research Council (ERC, contract 'dilute para-water', grant agreement number 339754), and the French "Equipements d'Excellence" Paris-en-Resonance, contract ANR-10-EQPX-09. The authors are grateful to Jim Kempf, Igor Koptyug and Malcolm Levitt for insightful comments.

\section{Supporting Information}

Simpson input file required to simulate deuterium spin-echo spectra with realistic pulses and coherence selection for an arbitrary spin temperature.

\section{References}

(1) Cox, S. F. J.; Bouffard, V.; Goldman, M. The Coupling of Two Nuclear Zeeman Reservoirs by the Electronic Spin-Spin Reservoir. J. Phys. C Solid State Phys. 1973, 6 (5), L100-L103.

(2) Abragam, A.; Goldman, M. Principles of Dynamic Nuclear Polarisation. Reports Prog. Phys. 1978, 41 (3), 395-467.

(3) Abragam A \& Goldman M. Nuclear Magnetism: Order and Disorder; Clarendon Press: Oxford, UK, 1982.

(4) Ardenkjaer-Larsen, J. H.; Fridlund, B.; Gram, A.; Hansson, G.; Hansson, L.; Lerche, M. H.; Servin, R.; Thaning, M.; Golman, K. Increase in Signal-to-Noise Ratio of \&gt; 10,000 Times in Liquid-State NMR. Proc. Natl. Acad. Sci. 2003, 100 (18), 10158-10163.

(5) Nelson, S. J.; Kurhanewicz, J.; Vigneron, D. B.; Larson, P. E. Z.; Harzstark, A. L.; Ferrone, M.; van Criekinge, M.; Chang, J. W.; Bok, R.; Park, I.; Reed, G.; Carvajal, L.; Small, E. J.; Munster, P.; Weinberg, V. K.; Ardenkjaer-Larsen, J. H.; Chen, A. P.; Hurd, R. E.; Odegardstuen, L.-I.; Robb, F. J.; Tropp, J.; Murray, J. A. Metabolic Imaging of Patients with Prostate Cancer Using Hyperpolarized [1-13C]Pyruvate. Sci. Transl. Med. 2013, 5 (198), 198ra108-198ra108.

(6) Perez-Linde, A. J. Application of Cross Polarisation Techniques to Dynamic Nuclear Polarisation Dissolution Experiments, Nottingham, 2009.

(7) Jannin, S.; Bornet, A.; Colombo, S.; Bodenhausen, G. Low-Temperature Cross Polarization in View of Enhancing Dissolution Dynamic Nuclear Polarization in NMR. Chem. Phys. Lett. 2011, 517 (4-6), 234-236. 
Spin Thermometry: a Straightforward Measure of Millikelvin Deuterium Spin Temperatures Achieved by Dynamic Nuclear Polarization

(8) Jannin, S.; Bornet, A.; Melzi, R.; Bodenhausen, G. High Field Dynamic Nuclear Polarization at 6.7 T: Carbon13 Polarization above 70\% within 20 Min. Chem. Phys. Lett. 2012, 549, 99-102.

(9) Bornet, A.; Melzi, R.; Jannin, S.; Bodenhausen, G. Cross Polarization for Dissolution Dynamic Nuclear Polarization Experiments at Readily Accessible Temperatures $1.2<\mathrm{T}<4.2 \mathrm{~K}$. Appl. Magn. Reson. 2012, 43 (1-2), 107-117.

(10) Bornet, A.; Melzi, R.; Perez Linde, A. J.; Hautle, P.; van den Brandt, B.; Jannin, S.; Bodenhausen, G. Boosting Dissolution Dynamic Nuclear Polarization by Cross Polarization. J. Phys. Chem. Lett. 2013, 4 (1), 111-114.

(11) Batel, M.; Däpp, A.; Hunkeler, A.; Meier, B. H.; Kozerke, S.; Ernst, M. Cross-Polarization for Dissolution Dynamic Nuclear Polarization. Phys. Chem. Chem. Phys. 2014, 16 (39), 21407-21416.

(12) Milani, J.; Vuichoud, B.; Bornet, A.; Miéville, P.; Mottier, R.; Jannin, S.; Bodenhausen, G. A Magnetic Tunnel to Shelter Hyperpolarized Fluids. Rev. Sci. Instrum. 2015, 86 (2).

Kiryutin, A. S.; Rodin, B. A.; Yurkovskaya, A. V.; Ivanov, K. L.; Kurzbach, D.; Jannin, S.; Guarin, D.; Abergel, D.; Bodenhausen, G. Transport of Hyperpolarized Samples in Dissolution-DNP Experiments. Phys. Chem. Chem. Phys. 2019, 21 (25), 13696-13705.

(14) Schäublin, S.; Höhener, A.; Ernst, R. R. Fourier Spectroscopy of Nonequilibrium States, Application to CIDNP, Overhauser Experiments and Relaxation Time Measurements. J. Magn. Reson. 1974, 13 (2), 196-216.

(15) Tropp, J. Multiplet Asymmetry and Multi-Spin Order in Liquid-State NMR Spectra of Hyperpolarized Compounds. Proc. Intl. Soc. Mag. Reson. Med. 2010, 18, 1026.

(16) Lau, J. Y. C.; Chen, A. P.; Gu, Y.-P.; Cunningham, C. H. A Calibration-Based Approach to Real-Time in Vivo Monitoring of Pyruvate $C_{1}$ and $C_{2}$ Polarization Using the $J$ cc Spectral Asymmetry. NMR Biomed. 2013,26 (10), 1233-1241.

(17) Vuichoud, B.; Milani, J.; Chappuis, Q.; Bornet, A.; Bodenhausen, G.; Jannin, S. Measuring Absolute Spin Polarization in Dissolution-DNP by Spin PolarimetrY Magnetic Resonance (SPY-MR). J. Magn. Reson. 2015, 260, 127-135.

(18) Meier, B.; Dumez, J.-N.; Stevanato, G.; Hill-Cousins, J. T.; Roy, S. S.; Håkansson, P.; Mamone, S.; Brown, R. C. D.; Pileio, G.; Levitt, M. H. Long-Lived Nuclear Spin States in Methyl Groups and Quantum-Rotor-Induced Polarization. J. Am. Chem. Soc. 2013, 135 (50), 18746-18749.

(19) Kuhns, P.; Gonen, O.; Waugh, J. S. Proton Spin-Spin and Spin-Lattice Relaxation in CaSO4 $\cdot$ XH2O below 1 K. J. Magn. Reson. 1989, 82 (2), 231-237.

(20) Mammoli, D.; Salvi, N.; Milani, J.; Buratto, R.; Bornet, A.; Sehgal, A. A.; Canet, E.; Pelupessy, P.; Carnevale, D.; Jannin, S.; Bodenhausen, G. Challenges in Preparing, Preserving and Detecting Para-Water in Bulk: Overcoming Proton Exchange and Other Hurdles. Phys. Chem. Chem. Phys. 2015, 17 (40), 26819-26827.

(21) Abragam, A.; Chapellier, M.; Jacquinot, J. F.; Goldman, M. Absorption Lineshape of Highly Polarized Nuclear Spin Systems. J. Magn. Reson. 1973, 10 (3), 322-346.

(22) Edwards, C. M.; Zhou, D.; Sullivan, N. S. Unusual Low-Temperature Effects on the NMR Line Shapes in Solid Hydrogen. Phys. Rev. B. Condens. Matter 1986, 34 (9), 6540-6542.

(23) Waugh, J. S.; Gonen, O.; Kuhns, P. Fourier Transform NMR at Low Temperatures. J. Chem. Phys. 1987, 86 (7), 3816-3818.

(24) Marohn, J. A.; Carson, P. J.; Hwang, J. Y.; Miller, M. A.; Shykind, D. N.; Weitekamp, D. P. Optical Larmor Beat 
Spin Thermometry: a Straightforward Measure of Millikelvin Deuterium Spin Temperatures Achieved by Dynamic Nuclear Polarization

Detection of High-Resolution Nuclear Magnetic Resonance in a Semiconductor Heterostructure. Phys. Rev. Lett. 1995, 75 (7), 1364-1367.

(25) Carnevale, D.; Ashbrook, S. E.; Bodenhausen, G. Solid-State NMR Measurements and DFT Calculations of the Magnetic Shielding Tensors of Protons of Water Trapped in Barium Chlorate Monohydrate. RSC Adv. 2014, 4 (99), 56248-56258.

(26) Carnevale, D.; Chinthalapalli, S.; Bodenhausen, G. Exciting Wide NMR Spectra of Static Solid Samples with Weak Radiofrequency Fields. Zeitschrift fur Phys. Chemie 2017, 231 (3), 527-543.

(27) Carnevale, D.; Pelupessy, P.; Bodenhausen, G. Cross-Term Splittings Due to the Orientational Inequivalence of Proton Magnetic Shielding Tensors: Do Water Molecules Trapped in Crystals Hop or Tunnel? J. Phys. Chem. Lett. 2019, 10 (12), 3224-3231.

(28) Carnevale, D.; Marhabaie, S.; Pelupessy, P.; Bodenhausen, G. Orientation-Dependent Proton Relaxation of Water Molecules Trapped in Solids: Crystallites with Long-Lived Magnetization. J. Phys. Chem. A 2019, 123 (45), 9763-9769.

(29) Aghelnejad, B.; Bodenhausen, G.; Marhabaie, S. A Low-Temperature Broadband NMR Probe for Multinuclear Cross-Polarization. ChemPhysChem 2019, 20 (21), 2830-2835.

(30) Kuzma, N. N.; Håkansson, P.; Pourfathi, M.; Ghosh, R. K.; Kara, H.; Kadlecek, S. J.; Pileio, G.; Levitt, M. H.; Rizi, R. R. Lineshape-Based Polarimetry of Dynamically-Polarized 15N 20 in Solid-State Mixtures. J. Magn. Reson. 2013, 234, 90-94.

(31) Sugishita, T.; Matsuki, Y.; Fujiwara, T. Absolute 1H Polarization Measurement with a Spin-Correlated Component of Magnetization by Hyperpolarized MAS-DNP Solid-State NMR. Solid State Nucl. Magn. Reson. 2019, 99, 20-26.

(32) Andersen, P. M.; Sullivan, N. S.; Rall, M.; Brison, J. P. Asymmetry of Field-Perturbed NQR Spectra: A New Ultra-Low Temperature Thermometer? Phys. B Condens. Matter 1991, 169 (1-4), 453-454.

(33) Chinthalapalli, S.; Bornet, A.; Carnevale, D.; Jannin, S.; Bodenhausen, G. Homonuclear Decoupling for Spectral Simplification of Carbon-13 Enriched Molecules in Solution-State NMR Enhanced by Dissolution DNP. Phys. Chem. Chem. Phys. 2016, 18 (16), 11480-11487.

(34) Long, J. R.; Ebelhäuser, R.; Griffin, R. G. ${ }^{2} \mathrm{H}$ NMR Line Shapes and Spin-Lattice Relaxation in $\mathrm{Ba}\left(\mathrm{ClO}_{3}\right) 2 .{ }^{2} \mathrm{H}$ 2 O. J. Phys. Chem. A 1997, 101 (6), 988-994.

(35) Sørensen, O. W.; Eich, G. W.; Levitt, M. H.; Bodenhausen, G.; Ernst, R. R. Product Operator Formalism for the Description of NMR Pulse Experiments. Progress in Nuclear Magnetic Resonance Spectroscopy. 1984, pp 163-192.

(36) Bodenhausen, G.; Freeman, R.; Turner, D. L. Suppression of Artifacts in Two-Dimensional J Spectroscopy. J. Magn. Reson. 1977, 27 (3), 511-514.

(37) Bodenhausen, G.; Kogler, H.; Ernst, R. R. Selection of Coherence-Transfer Pathways in NMR Pulse Experiments. J. Magn. Reson. 1984, 58 (3), 370-388.

(38) Carnevale, D.; Vitzthum, V.; Lafon, O.; Trébosc, J.; Amoureux, J. P.; Bodenhausen, G. Broadband Excitation in Solid-State NMR of Paramagnetic Samples Using Delays Alternating with Nutation for Tailored Excitation ('Para-DANTE'). Chem. Phys. Lett. 2012, 553, 68-76.

(39) Carnevale, D.; Perez Linde, A. J.; Bauer, G.; Bodenhausen, G. Solid-State Proton NMR of Paramagnetic Metal Complexes: DANTE Spin Echoes for Selective Excitation in Inhomogeneously Broadened Lines. Chem. Phys. 
Lett. 2013, 580, 172-178.

(40) Chan-Huot, M.; Wimperis, S.; Gervais, C.; Bodenhausen, G.; Duma, L. Deuterium MAS NMR Studies of Dynamics on Multiple Timescales: Histidine and Oxalic Acid. ChemPhysChem 2015, 16 (1), $204-215$.

(41) Guarin, D.; Marhabaie, S.; Rosso, A.; Abergel, D.; Bodenhausen, G.; Ivanov, K. L.; Kurzbach, D. Characterizing Thermal Mixing Dynamic Nuclear Polarization via Cross-Talk between Spin Reservoirs. J. Phys. Chem. Lett. 2017, 8 (22), 5531-5536.

(42) Jähnig, F.; Himmler, A.; Kwiatkowski, G.; Däpp, A.; Hunkeler, A.; Kozerke, S.; Ernst, M. A Spin-

Thermodynamic Approach to Characterize Spin Dynamics in TEMPO-Based Samples for Dissolution DNP at 7 T Field. J. Magn. Reson. 2019, 303, 91-104.

(43) Antonijevic, S.; Wimperis, S. Refocussing of Chemical and Paramagnetic Shift Anisotropies in 2H NMR Using the Quadrupolar-Echo Experiment. J. Magn. Reson. 2003, 164 (2), 343-350.

(44) Bak, M.; Rasmussen, J. T.; Nielsen, N. C. SIMPSON: A General Simulation Program for Solid-State NMR Spectroscopy. J. Magn. Reson. 2000, 147 (2), 296-330.

(45) Zaremba, S. K. Good Lattice Points, Discrepancy, and Numerical Integration. Ann. di Mat. Pura ed Appl. Ser. 4 1966, 73 (1), 293-317.

(46) Conroy, H. Molecular Schrödinger Equation. VIII. A New Method for the Evaluation of Multidimensional Integrals. J. Chem. Phys. 1967, 47 (12), 5307-5318.

(47) Cheng, V. B.; Suzukawa, H. H.; Wolfsberg, M. Investigations of a Nonrandom Numerical Method for Multidimensional Integration. J. Chem. Phys. 1973, 59 (8), 3992-3999. 
(a)

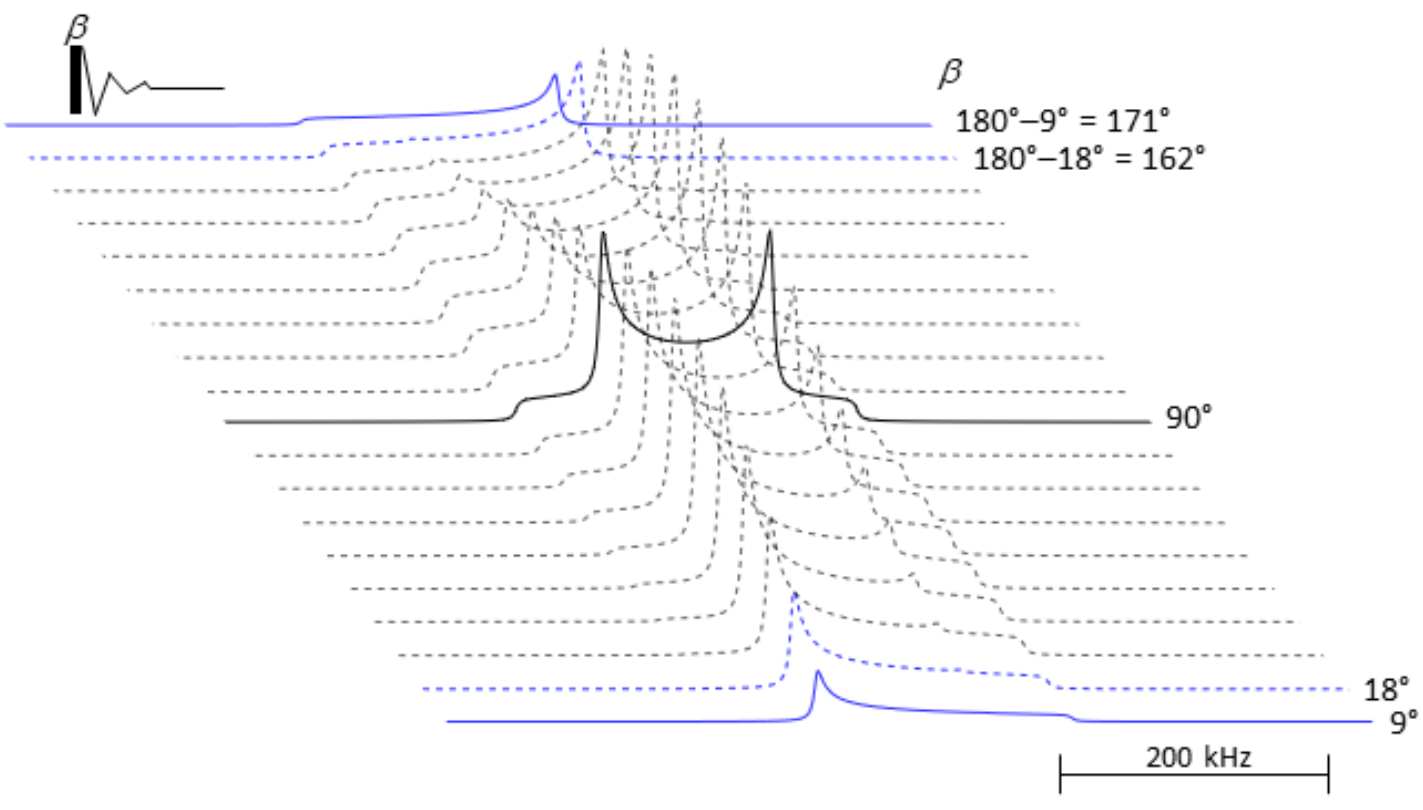

(b)

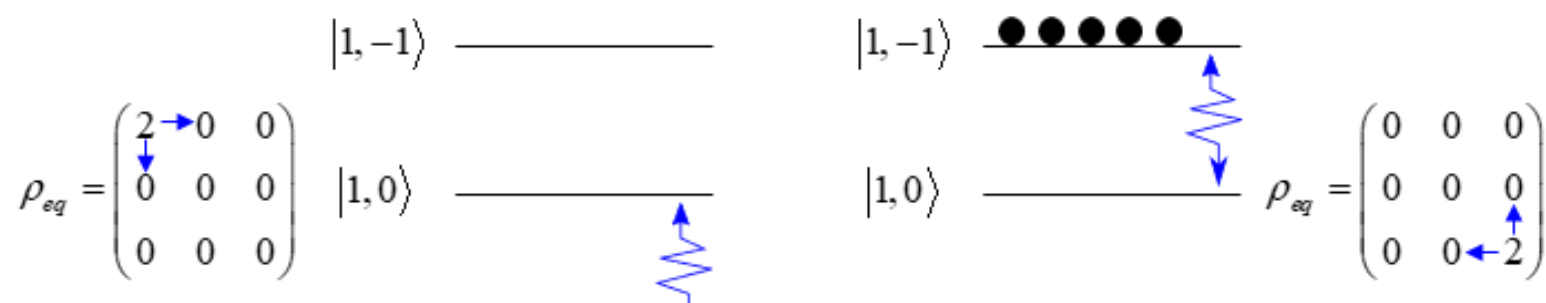

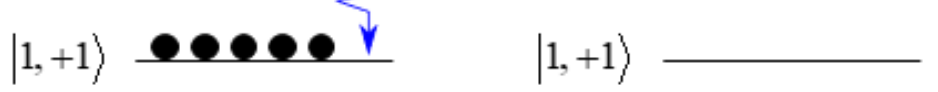

Figure 1 (a) Numerical simulations of first-order quadrupolar lineshapes of ${ }^{2} \mathrm{H}$ spins in a powder with isotropically distributed crystallites, obtained by Fourier transformation of simulated free induction decays (without any delay between excitation and observation) excited by a single ideal pulse applied in the center of the quadrupolar powder patterns, with variable nutation angles $\beta$ acting on a fully polarized initial state where only the ground state is populated $\left(T_{\text {spin }}\left({ }^{2} \mathrm{H}\right)=0 \mathrm{~K}\right)$. A quasi-pure singletransition spectrum is obtained for $\beta<20^{\circ}$, corresponding to one of the two 'lobes' or 'cusps' of the Pake pattern. For $160^{\circ}<\beta<180^{\circ}$, the other lobe is favored, while for $\beta=90^{\circ}$ one obtains a superposition of two symmetrical lobes. The relevant parameters were $C_{Q}=170 \mathrm{kHz}$ and $\eta_{Q}=0$, whereas the anisotropic chemical shift was neglected. (b) Density matrices and energy level diagrams for the bottom and top (blue) single-transition spectra of (a). 


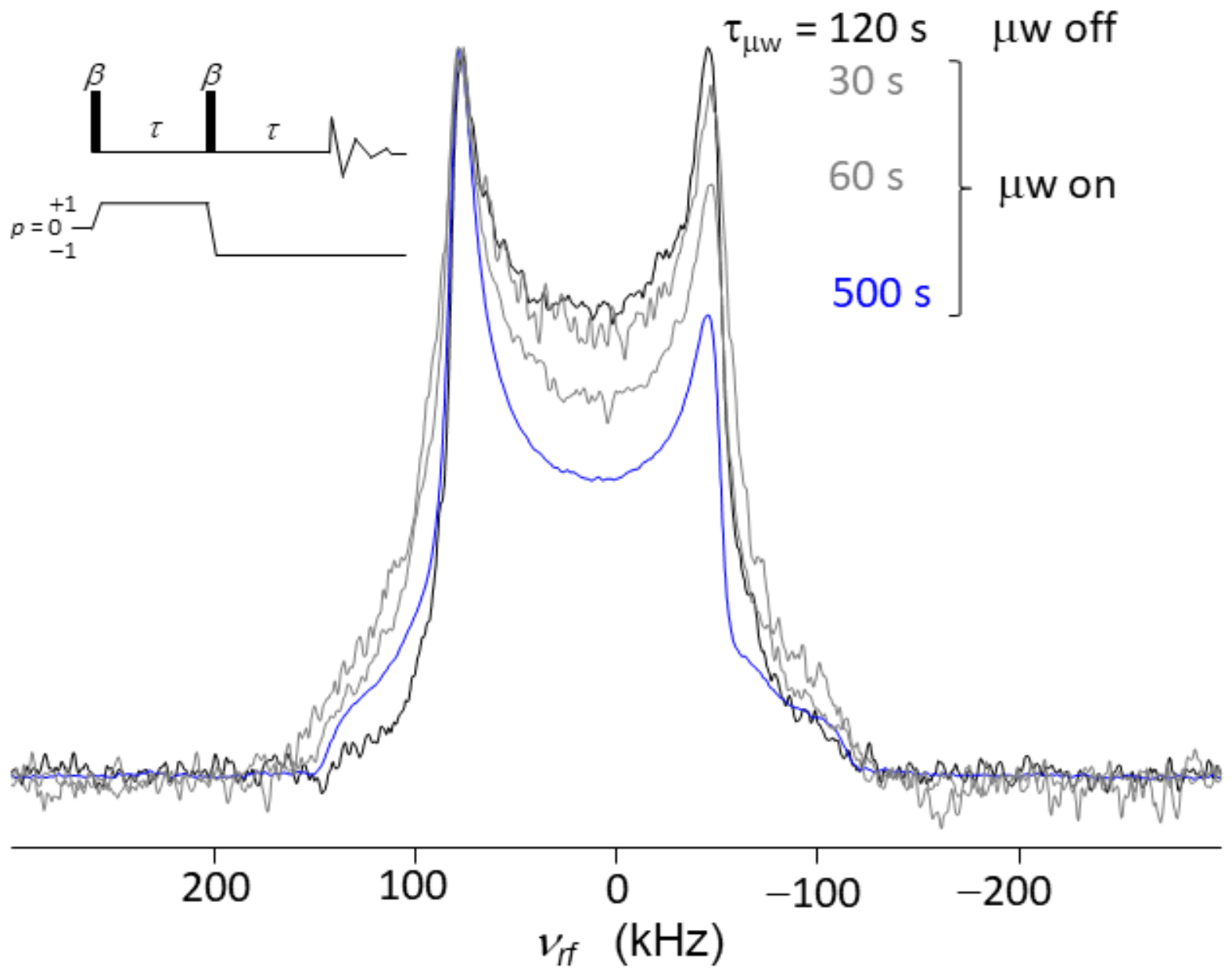

Figure 2 Experimental ${ }^{2} \mathrm{H}$ spectra of a sample consisting of $300 \mu \mathrm{l}$ of $60 \%$ ethanol- $\mathrm{d}_{6}, 30 \% \mathrm{D}_{2} \mathrm{O}$, $10 \% \mathrm{H}_{2} \mathrm{O}$ and $40 \mathrm{mM}$ TEMPOL, obtained with an exorcycled $\beta-\tau$ - $\beta$ - $\tau$ - quadrupolar echo with $\beta=18^{\circ}$ in a field $B_{0}=6.7 \mathrm{~T}$ where $v_{0}\left({ }^{1} \mathrm{H}\right)=285.3 \mathrm{MHz}$ and $v_{0}\left({ }^{2} \mathrm{H}\right)=43.8 \mathrm{MHz}$. After activating the microwave irradiation for a duration $\tau_{\mu \mathrm{w}}$, a progressively more pronounced asymmetry of the quadrupolar 'Pake pattern' is obtained as the polarization builds up. For sensitivity reasons, the black spectrum without microwave irradiation was acquired by averaging 16 echoes at $T_{\text {sample }}=4.0 \mathrm{~K}$ with $\beta=36^{\circ}$ ( $\left.\tau_{p}=2 \mu \mathrm{s}\right)$. All other spectra were acquired at $T_{\text {sample }}=1.3 \mathrm{~K}$ as a function of the duration of the microwave irradiation $\tau_{\mu \mathrm{w}}=30,60$ and $500 \mathrm{~s}$ by averaging only 4 phase-cycled echoes with $\beta=18^{\circ}\left(\tau_{p}=1 \mu \mathrm{s}\right)$. 


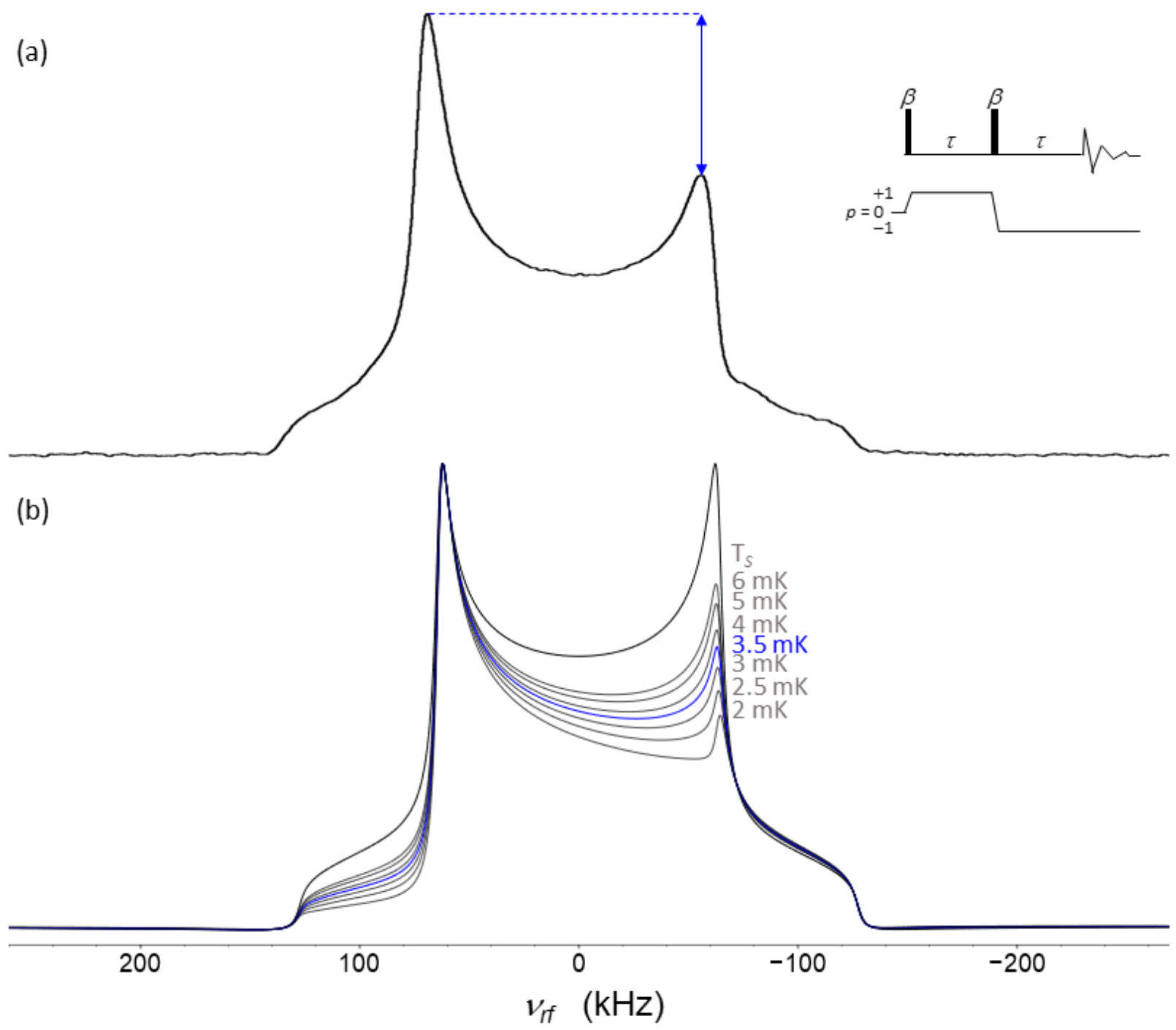

Figure $3 \quad$ (a) Hyperpolarized experimental ${ }^{2} \mathrm{H}$ spectrum shown in blue in Fig. 2. The asymmetry between the 'horns' of the quadrupolar 'Pake pattern' is highlighted by a blue arrow. (b) Numerical simulations assuming different spin temperatures $T_{\text {spin. }}$. The case that agrees best with the experiment of (a) is highlighted in blue, corresponding to $T_{\text {spin }}=3.5 \pm 0.5 \mathrm{mK}$ or $P\left(I_{z}\right)=29 \pm 4 \%$. 


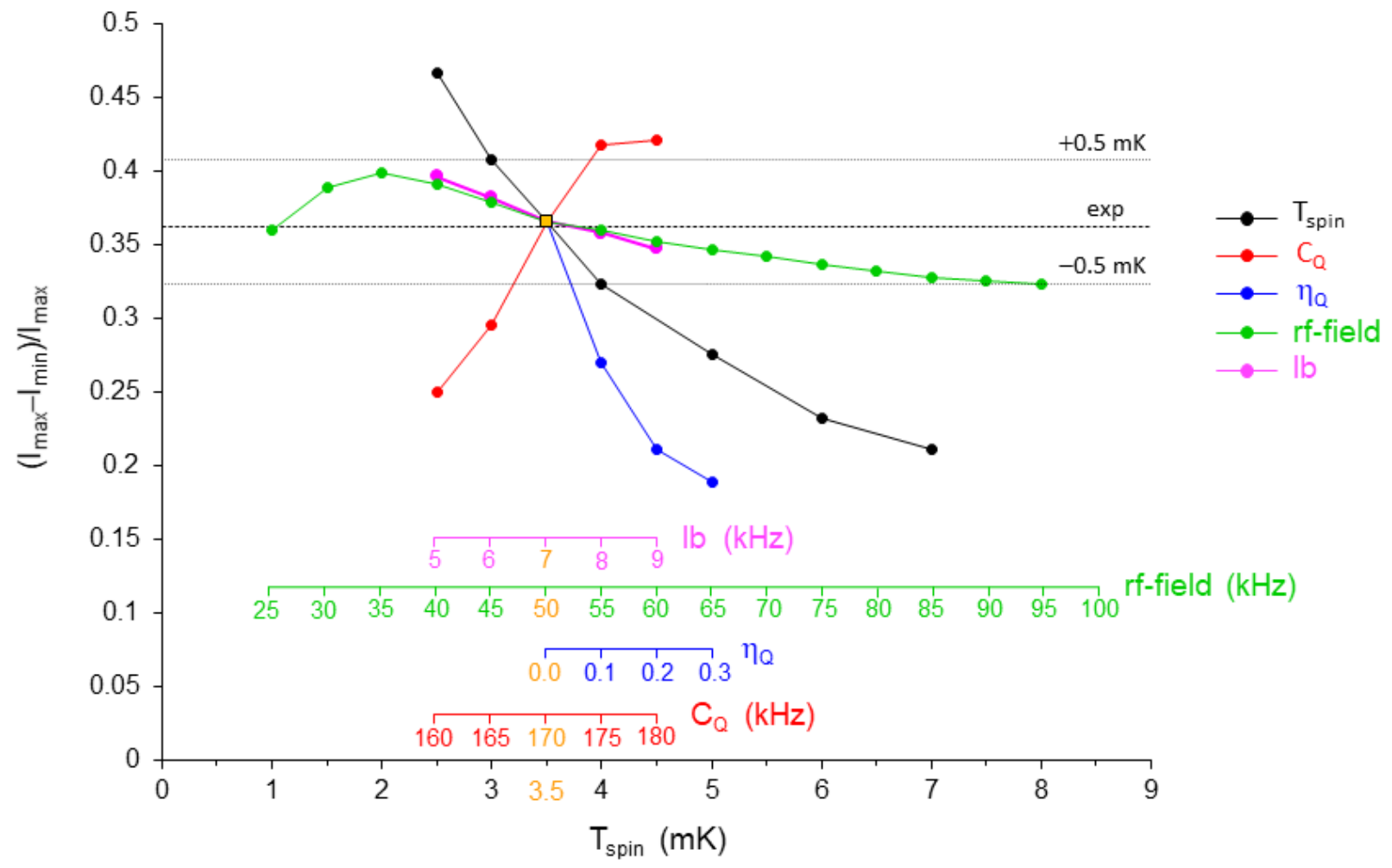

Figure $4 \quad$ Asymmetry of simulated hyperpolarized ${ }^{2} \mathrm{H}$ quadrupolar powder patterns expressed as $\left(I_{\max }-I_{\min }\right) / I_{\max }$, where $I_{\max }$ and $I_{\min }$ are the peak heights of the high- and low-frequency 'horns' of Pake patterns obtained by numerical simulations of $18^{\circ}-\tau-18^{\circ}-\tau$ - quadrupolar echoes combined with Exorcycle for different parameters. Powder averaging was performed over 4180 crystallite orientations and realistic rectangular pulses were taken into account. Black, red, blue, green and cyan data refer to the spin temperature $T_{\text {spin, }}$, the quadrupolar coupling constant $C_{Q}$, the asymmetry parameter $\eta_{Q}, r f$-field strength and homogeneous line broadening $l b$, respectively. The experimental value of the asymmetry seen in Fig. 3a is indicated by a horizontal dashed line. Thin dashed horizontal lines indicate an uncertainty interval of $\pm 0.5 \mathrm{mK}$. The optimal set of parameters is indicated in orange. 

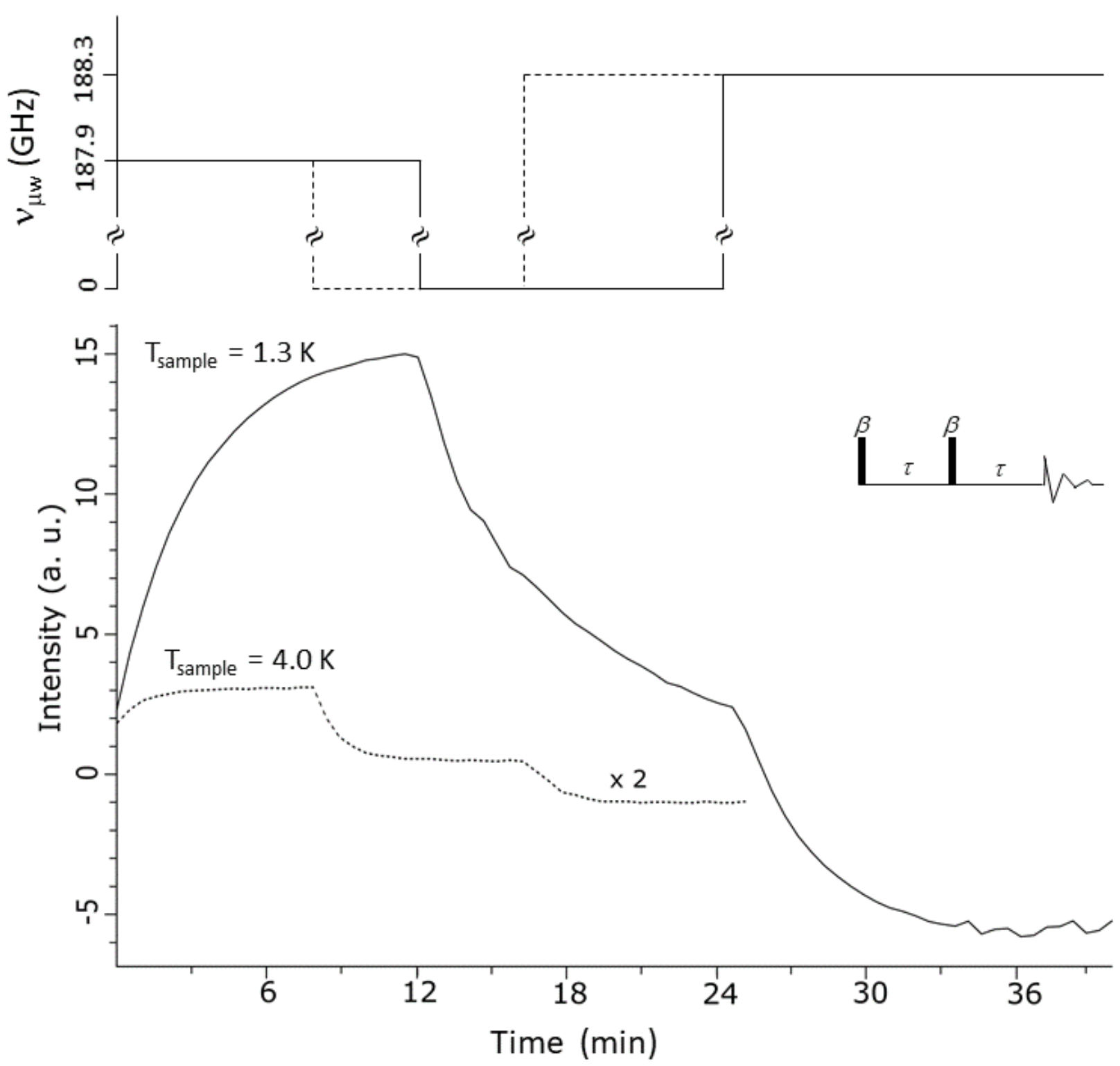

Figure $5 \quad$ Build-up of the integrals of ${ }^{2} \mathrm{H}$ spectra under the effect of microwave irradiation for $T_{\text {sample }}=1.3$ and $4.0 \mathrm{~K}$, shown by continuous and dashed lines, respectively. In all cases, after an initial presaturating train of pulses, a ${ }^{2} \mathrm{H}$ signal was acquired every $30 \mathrm{~s}$, excited by a single a $\beta$ - $\tau$ - $\beta$ - $\tau$ - echo (without phase cycling) with $\beta=27^{\circ}$ and $\tau=15 \mu \mathrm{s}$. The top panel indicates the corresponding microwave saturation frequencies at $v_{\mu \mathrm{w}}=187.9$ and $188.3 \mathrm{GHz}$, for positive and negative DNP enhancements, whereas $v_{\mu \mathrm{w}}=0$ indicates that the microwave source was turned off. The vertical scale of the data measured at $T_{\text {sample }}=4.0 \mathrm{~K}$ was amplified by a factor two. 


\section{For Table of Contents Only}
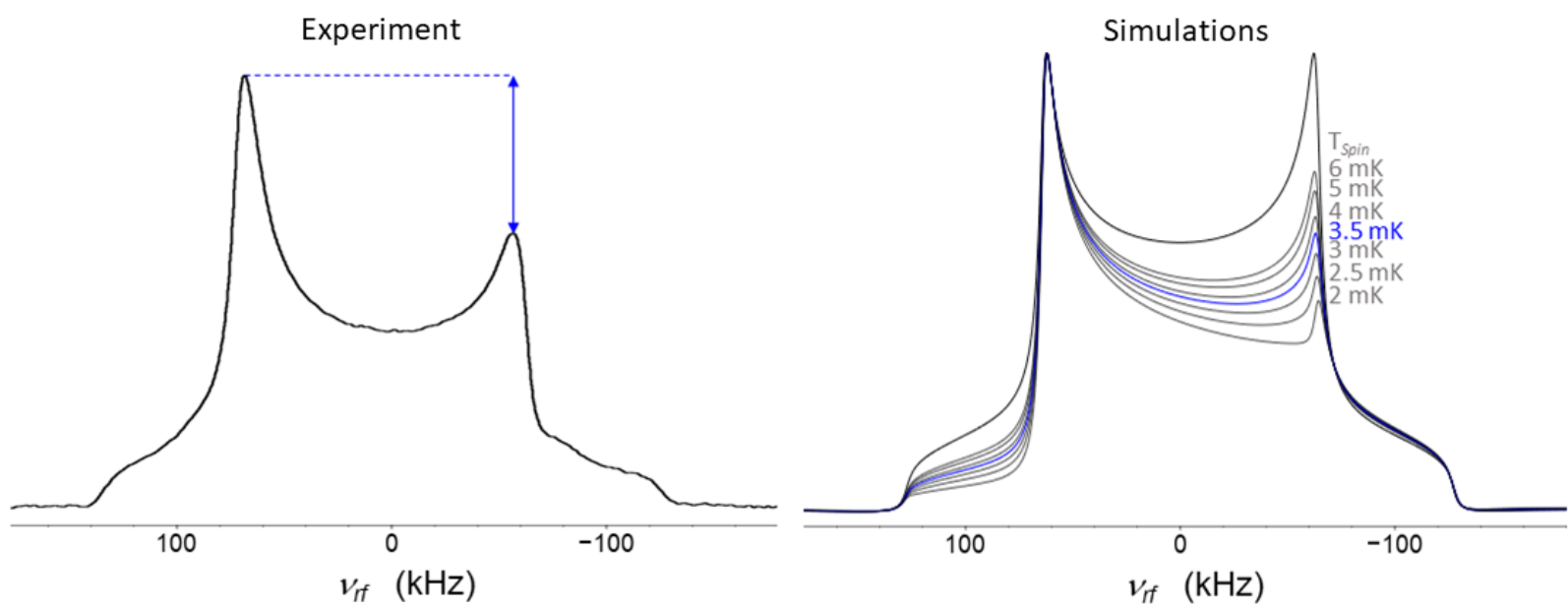